\title{
FUNDAMENTAL MATRIX FACTORIZATION IN THE FJRW-THEORY REVISITED
}

\author{
ALEXANDER POLISHCHUK
}

\begin{abstract}
We present an improved construction of the fundamental matrix factorization in the FJRW-theory given in [6]. The revised construction is coordinate-free and works for a possibly nonabelian finite group of symmetries. One of the new ingrediants is the category of dg-matrix factorizations over a dg-scheme.
\end{abstract}

\section{INTRODUCTION}

This short note is supposed to clarify the construction of the cohomological field theory associated with a quasihomogeneous polynomial $W$ and its finite group of symmetries $G$. Such a cohomological field theory, called the FJRW-theory was first proposed in [3]. Then, in [6] a different construction, based on categories of matrix factorizations, was given (conjecturally, the two constructions give the same cohomological field theory).

The approach of [6] is based on constructing certain fundamental matrix factorizations which live over the product of certain finite coverings of $\bar{M}_{g, n}$ (the moduli of $\Gamma$-spin structures) with affine spaces. It is this construction that we aim to clarify. More precisely, we would like to present the construction in such a way that it would be analogous to the construction of Ciocan-Fontanine and Kapranov of the virtual fundamental class in Gromov-Witten theory via dg-manifolds (see [1]). The second goal that we achieve is to present the construction without using coordinates on the vector space $V$ on which $W$ lives. This has an additional bonus that we can handle the case when the group $G$ is not necessarily commutative (but still finite).

The construction of [6] of the fundamental matrix factorization over $\mathcal{S} \times \prod_{i} V^{\gamma_{i}}$, where $\mathcal{S}$ is the moduli space of (rigidified) $\Gamma$-spin structures with some markings (see Sec. 3.1 for details) roughly has the following two steps. In Step 1 one considers the object $R \pi_{*}(\mathcal{V})$ in the derived category $D(\mathcal{S})$, where $\pi: \mathcal{C} \rightarrow \mathcal{S}$ is the universal curve, $\mathcal{V}$ is the underlying vector bundle of the universal $\Gamma$-spin structure, and then equips it with some additional structure. In Step 2 one realizes $R \pi_{*}(\mathcal{V})$ by a 2 -term complex $[A \rightarrow B]$, such that there is a morphism

$$
Z: X=\operatorname{tot}(A) \rightarrow \prod_{i} V^{\gamma_{i}}
$$

and a Koszul matrix factorization of $Z^{*}\left(\sum W_{i}\right)$, where $W_{i}=\left.W\right|_{V^{\gamma_{i}}}$. Then the fundamental matrix factorization is obtained by taking its push-forward with respect to the morphism $(p, Z): X \rightarrow \mathcal{S} \times V^{\gamma_{i}}$, where $p: X \rightarrow \mathcal{S}$ is the projection. Note that here the space $X$ is non-canonical, so one has to check independence on the choices made.

Supported in part by the NSF grant DMS-1700642 and by the Russian Academic Excellence Project ' $5-100$ '. 
The main idea of the present paper is to change the conceptual framework slightly by observing that in fact one gets a dg-matrix factorization on a $d g$-scheme over $\mathcal{S} \times \prod_{i} V^{\gamma_{i}}$ (the terminology is explained in Sec. 1). Namely, for a non-negatively graded complex of vector bundles $C^{\bullet}$ over $\mathcal{S}$, one can define the corresponding dg-scheme over $\mathcal{S}$,

$$
\left[C^{\bullet}\right]:=\operatorname{Spec}\left(S^{\bullet}\left(C^{\bullet}\right)^{\vee}\right) \text {. }
$$

In our case we consider the dg-scheme

$$
\mathcal{X}:=\left[R \pi_{*}(\mathcal{V})\right]
$$

More concretely, if we realize $\mathcal{V}$ by a 2 -term complex $\mathcal{V}=[A \rightarrow B]$ then our dg-scheme is realized by the sheaf of dg-algebras

$$
\mathcal{O}_{\mathcal{X},[A \rightarrow B]}:=S^{\bullet}\left(B^{\vee} \rightarrow A^{\vee}\right) .
$$

Then we interpret the additional structure on $R \pi_{*}(\mathcal{V})$ coming from the universal $\Gamma$-spin structure as a structure of a dg-matrix factorization on the structure sheaf of $\mathcal{X}$. More precisely, we get a morphism

$$
Z_{\mathcal{X}}: \mathcal{X} \rightarrow \prod_{i} V^{\gamma_{i}}
$$

and a function of degree $-1, f_{-1} \in \mathcal{O}_{\mathcal{X},[A \rightarrow B]}^{-1}$, such that

$$
d\left(f_{-1}\right)=-Z_{\mathcal{X}}^{*}\left(\sum W_{i}\right)
$$

Now the fundamental matrix factorization is obtained as the push-forward of $\left(\mathcal{O}_{\mathcal{X}}, d+\right.$ $f_{-1} \cdot$ id) with respect to the morphism $\mathcal{X} \rightarrow \mathcal{S} \times \prod_{i} V^{\gamma_{i}}$.

The connection with the original approach is the following: for each presentation $\mathcal{V}=$ $[A \rightarrow B]$, for which the first construction works, there is a morphism $q: \mathcal{X} \rightarrow X=\operatorname{tot}(A)$, such that $Z \circ q=Z_{\mathcal{X}}$, and an isomorphism of the push-forward $q_{*}\left(\mathcal{O}_{\mathcal{X}}, d+f_{-1} \cdot\right.$ id $)$ with the Koszul matrix factorization of $Z^{*}\left(\sum W_{i}\right)$ constructed through the first approach.

The second technical improvement we present is in the construction of $f_{-1}$. The idea is to work systematically with the categories of sheaves over pairs (scheme, closed subscheme) to deal with non-functoriality of the cone construction (such categories fit into the framework of Lunts's poset schemes in [5]). Namely, we work with the enhancement of the usual push-forward with respect to the projection $\pi: \mathcal{C} \rightarrow \mathcal{S}$ to a morphism of pairs $(\mathcal{C}, \Sigma) \rightarrow(\mathcal{S}, \mathcal{S})$, where $\Sigma \subset \mathcal{C}$ is the union of the images of the universal marked points (see Sec. 2).

Throughout this work the ground field is $\mathbb{C}$.

Acknowledgments. I am grateful to Felix Janda and Yongbin Ruan for organizing the RTG Conference on Witten's $r$-spin class and related topics in January 2017, where the results of this note were first presented. I also thank Institut Mathematique Jussieu and Institut des Hautes Etudes Scientifiques for hospitality and excellent working conditions during preparation of this paper.

\section{Matrix FACtORIZATiOns OVER DG-SCHEMES}

1.1. Definition. We consider dg-schemes in the spirit of [1]. We fix a space $S$ (a scheme or a stack), and consider the structure sheaf of a dg-scheme over $S$ to be a sheaf $\left(\mathcal{O}_{X}^{\bullet}, d\right)$ 
of $\mathbb{Z}_{-}$-graded commutative dg-algebras over $\mathcal{O}_{S}$ (one can make a restriction $\mathcal{O}_{X}^{0}=\mathcal{O}_{S}$, but it is not really necessary).

Given a function $f_{0} \in \mathcal{O}_{X}^{0}$ we can consider the category of (quasicoherent) dg-matrix factorizations of $f_{0}$. By definition, these are $\mathbb{Z} / 2$-graded complexes of sheaves $P=P^{\overline{0}} \oplus P^{\overline{1}}$ together with a (quasicoherent) $\mathcal{O}_{X}^{\sharp}$-module structure, such that $\mathcal{O}_{X}^{i} \cdot P^{\bar{a}} \subset P^{\overline{i+a}}$. In addition $P$ is equipped with an odd differential $\delta$ satisfying the Leibnitz identity

$$
\delta(\phi \cdot p)=d(\phi) \cdot p+(-1)^{k} \phi \delta(p),
$$

for $\phi \in \mathcal{O}_{X}^{k}, p \in P$, and the equation $\delta^{2}=f_{0} \cdot \operatorname{id}_{P}$.

Example 1.1.1. Given an element $f_{-1} \in \mathcal{O}_{X}^{-1}$, such that $d\left(f_{-1}\right)=f_{0}$, we get a structure of a dg-matrix factorization on $\mathcal{O}_{X}^{\bullet}$ by setting

$$
\delta(\phi)=d(\phi)+f_{-1} \cdot \phi .
$$

(In checking that $\delta^{2}=0$ one has to use the fact that $f_{-1}^{2}=0$.)

The above example can be obtained from the following more general operation. Suppose we are given a function $f_{0} \in \mathcal{O}_{X}^{0}$ and a dg-matrix factorization $(P, \delta)$ of $f_{0}$. Then for any $f_{-1} \in \mathcal{O}_{X}^{0}$ we can change the differential $\delta$ to $\delta+f_{-1} \cdot \operatorname{id}_{P}$. Then $\left(P, \delta+f_{-1} \cdot \mathrm{id}_{P}\right)$ will be a dg-matrix factorization of $f_{0}+d\left(f_{-1}\right)$.

1.2. Positselski's framework of quasicoherent CDG-algebras. More generally, we can assume that $f_{0}$ a section in $\mathcal{O}_{X}^{0} \otimes L$, where $L$ is a locally free $\mathcal{O}_{X}^{0}$-module of rank 1. The theory of the corresponding categories of dg-matrix factorizations fits into the framework of quasicoherent CDG-algebras developed by Positselski (see [2, Sec. 1]).

With the data $\left(\mathcal{O}_{X}^{\bullet}, L, f_{0}\right)$ as above we can associate a quasicoherent CDG-algebra

$$
\mathcal{B}:=\bigoplus_{n \in \mathbb{Z}} \mathcal{O}_{X}^{\bullet} \otimes_{\mathcal{O}_{X}^{0}} L^{\otimes n}[-2 n]
$$

with the natural structure of a complex of sheaves (i.e., the $\mathbb{Z}$-grading and the differential $d$ ), the natural product and the global curvature element given by $f_{0} \in \mathcal{O}^{0} \otimes L \subset \mathcal{B}_{2}$.

Now a quasicoherent dg-matrix factorization is a quasicoherent DG-module over $\mathcal{B}$, i.e., a graded $\mathcal{B}$-module $M=\bigoplus_{n} M_{n}$, equipped with a differential $\delta=\delta_{M}$ such that $\delta^{2}=f_{0} \cdot \operatorname{id}_{M}$ and $\delta$ satisfies the Leibnitz identity with respect to the $\mathcal{B}$-action. Note that such a DG-module necessarily has

$$
M_{n+2} \simeq M_{n} \otimes L
$$

so it is determined by the components $M_{0}$ and $M_{1}$, and we get the structure of a dg-matrix factorization on $M_{0} \oplus M_{1}$.

There are several exotic derived categories associated to a quasicoherent CDG-algebra. The one that is most relevant for the theory of dg-matrix factorizations is the category

$$
\mathrm{qcoh}-\operatorname{MF}_{f f d}\left(f_{0}\right):=D^{c o}\left(\mathcal{B}-\mathrm{qcoh}_{f f d}\right) \simeq D^{c o}\left(\mathcal{B}-\mathrm{qcoh}_{f l}\right) \simeq D^{a b s}\left(\mathcal{B}-\mathrm{qcoh}_{f l}\right),
$$

where the superscripts "abs" and "co" refer to "absolute" and "coderived", while the subscripts "fl" and "ffd" mean "flat" and "finite flat dimension" (see [2, Sec. 1]). 
Assume that $f:\left(X, \mathcal{O}_{X}^{\bullet}\right) \rightarrow\left(Y, \mathcal{O}_{Y}^{\bullet}\right)$ is a morphism of finite flat dimension, $L$ is a locally free $\mathcal{O}_{Y}^{0}$-module of rank $1, W_{0}$ is a section of $L$. Then we have the induced section $f^{*} W_{0}$ of $f^{*} L$. In this situation we have the push-forward functor (see [2, Prop. 1.9])

$$
R f_{*}: \mathrm{qcoh}-\mathrm{MF}_{f f d}\left(f^{*} W_{0}\right) \rightarrow \mathrm{qcoh}-\mathrm{MF}_{f f d}\left(W_{0}\right) .
$$

1.3. Koszul matrix factorizations as push-forwards. Let $V$ be a vector bundle over $X$, and suppose we have sections $\alpha \in H^{0}\left(X, V^{\vee}\right), \beta \in H^{0}(X, V)$. With these data one associates a Koszul matrix factorization $\{\alpha, \beta\}$ of $W=\langle\alpha, \beta\rangle$, whose underlying super-vector bundle is $\Lambda^{\bullet}(V)$. On the other hand, we have the derived zero locus of $\beta$, $\mathcal{Z}(\beta) \rightarrow X$, which corresponds to the dg-algebra given by the Koszul complex of $\beta$ :

$$
\mathcal{O}_{\mathcal{Z}(\beta)}=\left(\bigwedge^{\bullet}(V), d=\iota_{\beta}\right)
$$

Now we can view $\alpha$ as a function of degree -1 on $\mathcal{Z}(\beta)$ such that $d(\alpha)$ is the pull-back of $W$. Thus, by definition, $\{\alpha, \beta\}$ is the push-forward of the dg-matrix factorization $\left(\mathcal{O}_{\mathcal{Z}(\beta)}, d+\alpha \cdot \mathrm{id}\right)$.

This explains why in the case when $\beta$ is a regular section of $V$, the Koszul matrix factorization $\{\alpha, \beta\}$ is equivalent to the push-forward of the structure sheaf on the usual zero locus of $\beta$.

\section{TRACE MAPS VIA MORPHISMS OF PAIRS}

2.1. Sheaves on pairs. Let $\iota: Y \rightarrow X$ be a closed embedding.

We consider a very simple poset scheme in the sense of [5] for the poset consisting of two elements $\alpha>\beta$, so that $X_{\alpha}=Y$ and $X_{\beta}=X$. Then a quasicoherent sheaf on this poset scheme is a triple $\left(\mathcal{F}_{\alpha}, \mathcal{F}_{\beta}, \phi\right)$, with $\mathcal{F}_{\alpha} \in \operatorname{Qcoh}(Y), \mathcal{F}_{\beta} \in \mathrm{Q} \operatorname{coh}(X)$ and $\phi: \mathcal{F}_{\beta} \rightarrow \iota_{*} \mathcal{F}_{\alpha}$ is a morphism. We denote by $\mathrm{Q} \operatorname{coh}(X, Y)$ this abelian category, and by $\operatorname{Coh}(X, Y)$ its subcategory corresponding to $\mathcal{F}_{\alpha} \in \operatorname{Coh}(Y), \mathcal{F}_{\beta} \in \operatorname{Coh}(X)$.

Note that the derived category $D^{b} \operatorname{Coh}(X, Y)$ has a natural monoidal structure given by the tensor product, so we can also define symmetric powers of objects in $D^{b} \operatorname{Coh}(X, Y)$.

Given a morphism of pairs $f:(X, Y) \rightarrow\left(X^{\prime}, Y^{\prime}\right)$ we have a natural derived pushforward morphism

$$
R f_{*}: D^{+} \mathrm{Q} \operatorname{coh}(X, Y) \rightarrow D^{+} \mathrm{Q} \operatorname{coh}\left(X^{\prime}, Y^{\prime}\right) .
$$

The push-forward is compatible with the tensor products in the usual way: we have natural morphisms

$$
R f_{*}(F) \otimes R f_{*}(G) \rightarrow R f_{*}(F \otimes G), \quad S^{\bullet} R f_{*}(F) \rightarrow R f_{*} S^{\bullet}(F) .
$$

We have a fully faithful exact embedding $j_{!}: D \mathrm{Q} \operatorname{coh}(X) \rightarrow D \mathrm{Q} \operatorname{coh}(X, Y)$ sending $\mathcal{G}$ to $\mathcal{F}_{\alpha}=0, \mathcal{F}_{\beta}=\mathcal{G}$. There is a right adjoint functor to it (see [5]),

$$
R j^{!}: D^{+} \mathrm{Qcoh}(X, Y) \rightarrow D^{+} \mathrm{Qcoh}(X),
$$

which is defined as the right derived functor of the functor

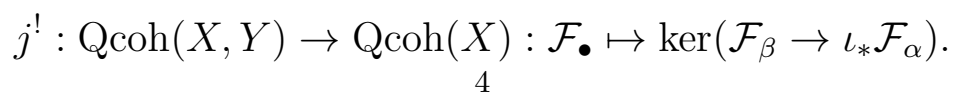


Note that objects $\mathcal{F}_{\bullet} \in \mathrm{Q} \operatorname{coh}(X, Y)$, such that $\mathcal{F}_{\beta} \rightarrow \iota_{*} \mathcal{F}_{\alpha}$ is surjective, are acyclic with respect to $j$ !. Furthermore, every object of $\mathrm{Q} \operatorname{coh}(X, Y)$ has a canonical resolutions by such acyclic objects:

$$
0 \rightarrow\left(\mathcal{F}_{\alpha}, \mathcal{F}_{\beta}\right) \rightarrow\left(\mathcal{F}_{\alpha}, \mathcal{F}_{\beta} \oplus \iota_{*} \mathcal{F}_{\alpha}\right) \rightarrow\left(0, \iota_{*} \mathcal{F}_{\alpha}\right) \rightarrow 0
$$

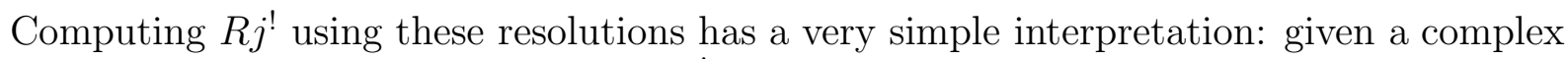
$\left(\mathcal{F}_{\alpha}^{\bullet}, \mathcal{F}_{\beta}^{\bullet}\right)$ over Qcoh $(X)$, the functor $R j^{!}$sends it to the complex

$$
\operatorname{Cone}\left(\mathcal{F}_{\beta}^{\bullet} \rightarrow \iota_{*} \mathcal{F}_{\alpha}^{\bullet}\right)[-1] \text {. }
$$

In particular, there is a natural exact triangle

$$
R j^{!}\left(\mathcal{F}_{\alpha}^{\bullet}, \mathcal{F}_{\beta}^{\bullet}\right) \rightarrow \mathcal{F}_{\beta}^{\bullet} \rightarrow \iota_{*} \mathcal{F} \bullet_{\alpha} \rightarrow \ldots
$$

We also have the following compatibility between $R j^{!}$and the push-forward.

Lemma 2.1.1. Let $f:(X, Y) \rightarrow\left(X^{\prime}, Y^{\prime}\right)$ be a morphism of pairs. Assume that there exists a finite open covering of $X$, affine over $X^{\prime}$. Then for $\mathcal{F} \in D^{+} \mathrm{Q} \operatorname{coh}(X, Y)$ we have a natural isomorphism

$$
R j^{!} R f_{*}(\mathcal{F}) \simeq R f_{*} R j^{!}(\mathcal{F})
$$

in $D^{+} \mathrm{Q} \operatorname{coh}\left(X^{\prime}\right)$.

Proof. Let us choose a quasi-isomorphism $\mathcal{F} \rightarrow \widetilde{\mathcal{F}}$, such that all $\widetilde{\mathcal{F}}_{\alpha}^{i}$ and $\widetilde{\mathcal{F}}_{\beta}^{i}$ are $f_{*}$-acyclic (this can be done using Cech resolutions). Then the left-hand side of (2.1.2) is represented by the complex

$$
\operatorname{Cone}\left(f_{*} \widetilde{\mathcal{F}}_{\beta} \rightarrow \iota_{*} f_{*} \widetilde{\mathcal{F}}_{\alpha}\right)[-1] \text {. }
$$

On the other hand, the terms of $\operatorname{Cone}\left(\widetilde{\mathcal{F}}_{\beta} \rightarrow \iota_{*} \widetilde{\mathcal{F}}_{\alpha}\right)[-1]$ are also $f_{*}$-acyclic, so the righthand side of (2.1.2) is represented by the complex

$$
f_{*} \operatorname{Cone}\left(\widetilde{\mathcal{F}}_{\beta} \rightarrow \iota_{*} \widetilde{\mathcal{F}}_{\alpha}\right)[-1],
$$

which is isomorphic to the one above.

2.2. Differentials on curves. Let $\pi: \mathcal{C} \rightarrow \mathcal{S}$ be a family of stable curves, $p_{i}: \mathcal{S} \rightarrow$ $\mathcal{C}, i=1, \ldots, r$, be sections of $\pi$, such that $\pi$ is smooth along their images, and let $\Sigma=\sqcup_{i} p_{i}(\mathcal{S})$. We view $(\mathcal{C}, \Sigma)$ as a poset scheme and consider the corresponding category $\operatorname{Coh}(\mathcal{C}, \Sigma)$ whose objects are collections $\left(F,\left(F_{i}\right),\left(f_{i}\right)\right)$, where $F$ is a coherent sheaf on $\mathcal{C}$, $F_{i}$ is a coherent sheaf on $\mathcal{S}$ and $f_{i}: F \rightarrow p_{i *} F_{i}$ is a morphism. Sometimes we will omit the morphisms $\left(f_{i}\right)$ from the notation and just write $\left(F,\left(F_{i}\right)\right)$.

Set $\omega_{\mathcal{C} / \mathcal{S}}^{\log }=\omega_{\mathcal{C} / \mathcal{S}}(\Sigma)$. Recall that we have natural residue maps

$$
\operatorname{Res}_{\Sigma}:\left.\omega_{\mathcal{C} / \mathcal{S}}^{\log }\right|_{\Sigma} \stackrel{\sim}{\longrightarrow} \mathcal{O}_{\Sigma}
$$

so that $\operatorname{ker}\left(\operatorname{Res}_{\Sigma}\right)$ is identified with $\omega_{\mathcal{C} / \mathcal{S}}$. Thus, we can view the triple

$$
\left[\omega_{\mathcal{C} / \mathcal{S}}^{\log }, \Sigma\right]:=\left(\omega_{\mathcal{C} / \mathcal{S}}^{\log }, \mathcal{O}_{\Sigma}, \operatorname{Res}_{\Sigma}\right)
$$

as an object of the category $\operatorname{Coh}(\mathcal{C}, \Sigma)$. Furthermore, we have

$$
R j^{!}\left[\omega_{\mathcal{C} / \mathcal{S}}^{\log }, \Sigma\right] \simeq \omega_{5} / \mathcal{S}
$$


Note that we have a morphism of pairs

$$
\pi:(\mathcal{C}, \Sigma) \rightarrow(\mathcal{S}, \mathcal{S})
$$

By Lemma 2.1.1, the object $R \pi_{*}\left[\omega_{\mathcal{C} / \mathcal{S}}^{\log }, \Sigma\right]$ satisfies

$$
R j^{!} R \pi_{*}\left[\omega_{\mathcal{C} / \mathcal{S}}^{\log }, \Sigma\right] \simeq R \pi_{*} \omega_{\mathcal{C} / \mathcal{S}} .
$$

Note also that we have a morphism of exact triangles (which will be used later)

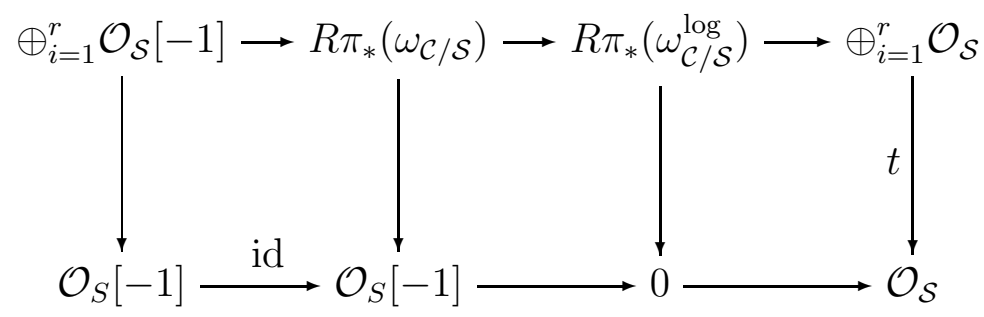

where $t$ is given by the summation.

The above constructions also work in the case of a family of orbicurves with stable coarse moduli spaces.

\section{Fundamental matrix Factorization}

3.1. Setup and the moduli spaces of $\Gamma$-spin structures. Let us recall the setup of the FJRW theory (see [3], [6]), or rather its slight generalization to noncommutative finite groups of symmetries (as in [4]).

We start with a finite-dimensional vector space $V$ equipped with an effective $\mathbb{G}_{m}$-action called the $R$-charge, such that all the weights of this action on $V$ are positive. We denote the corresponding subgroup in $\operatorname{GL}(V)$ by $\mathbb{G}_{m, R}$. and let $W$ be a function of weight $d$ on $V$. Also, we fix a finite subgroup $G \subset \mathrm{GL}(V)$ such that $W$ is $G$-invariant, $G$ commutes with $\mathbb{G}_{m, R}$ and $G$ contains a fixed element $J \in \mathbb{G}_{m, R}$ of order $d$.

We define $\Gamma \subset \mathrm{GL}(V)$ to be the algebraic subgroup generated by $G$ and by $\mathbb{G}_{m, R}$. There is a canonical exact sequence

$$
1 \rightarrow G \rightarrow \Gamma \stackrel{\chi}{\longrightarrow} \mathbb{G}_{m} \rightarrow 1,
$$

where $\chi$ restricts to the subgroup $\mathbb{G}_{m, R}$ as $\lambda \mapsto \lambda^{d}$.

As in [6], we consider the moduli space of $\Gamma$-spin structures: it classifies stable orbicurves $\left(C, p_{1}, \ldots, p_{n}\right)$ equipped with $\Gamma$-principal bundle $P$ (our convention is that we have a right action of $\Gamma$ on $P$ ), together with an isomorphism $\chi_{*} P \stackrel{\sim}{\longrightarrow} \omega_{C}^{\log } \backslash 0$. We can think of the latter isomorphism as a morphism $\chi_{P}: P \rightarrow \omega_{C}^{\log } \backslash 0$ satisfying

$$
\chi_{P}(x \gamma)=\chi(\gamma) \cdot \chi_{P}(x)
$$

for $\gamma \in \Gamma$.

In addition to requiring the coarse moduli of $C$ to be Deligne-Mumford stable, we require that for each marked point $p_{i}$ the morphism $B \operatorname{Aut}\left(p_{i}\right) \rightarrow B \Gamma$ induced by $P$ is representable. By looking at the corresponding embedding $\operatorname{Aut}\left(p_{i}\right) \simeq \mathbb{Z} / m_{i} \rightarrow \Gamma$ defined up to a conjugacy, we get a conjugacy class $\gamma_{i}$ in $\Gamma$. Thus, we get a decomposition of our moduli stack into a disjoint union of open and closed substacks $\mathcal{S}_{g}\left(\gamma_{1}, \ldots, \gamma_{n}\right)$. As in $[3$, 
Sec. 2.2], one shows that these are smooth and proper DM stacks with projective coarse moduli.

Let $\pi: \mathcal{C} \rightarrow \mathcal{S}_{g}\left(\gamma_{1}, \ldots, \gamma_{i}\right)$ be the universal curve over $\mathcal{S}_{g}\left(\gamma_{1}, \ldots, \gamma_{n}\right)$, and let $\mathcal{V}=$ $\mathcal{P} \times_{\Gamma} V$ be the vector bundle over $\mathcal{C}$ associated with the universal $\Gamma$-spin structure $\mathcal{P}$ via the embedding $\Gamma \subset \mathrm{GL}(V)$. Note that $\mathcal{V}$ is equipped with a $\mathbb{G}_{m, R^{-a c t i o n}}$ (through its action on $V$ ).

As in [6], we also consider a Galois covering $\mathcal{S}_{g}^{\text {rig }}\left(\gamma_{1}, \ldots, \gamma_{n}\right) \rightarrow \mathcal{S}_{g}\left(\gamma_{1}, \ldots, \gamma_{n}\right)$ corresponding to choices of a rigidification at every marked point. A rigidification is an isomorphism of the restriction of $P$ to $p_{i} / \operatorname{Aut}\left(p_{i}\right) \simeq B\left\langle\gamma_{i}\right\rangle$ with $V /\left\langle\gamma_{i}\right\rangle$ (viewed as a bundle over $B\left\langle\gamma_{i}\right\rangle$. There is a natural simply transitive action of the group $\prod_{i} C_{G}\left(\gamma_{i}\right)$ on the set of rigidifications at $p_{1}, \ldots, p_{n}$, where $C_{G}(\gamma) \subset G$ is the centralizer of $\gamma \in G$.

3.2. Construction. Let us set for now $\mathcal{S}=\mathcal{S}_{g}^{\text {rig }}\left(\gamma_{1}, \ldots, \gamma_{n}\right)$ and consider the pull-back of all the objects to $\mathcal{S}$ (denoting them by the same symbols).

Note that we have a natural projection $V /\left\langle\gamma_{i}\right\rangle \rightarrow V^{\gamma_{i}}$. Thus, from rigidification structures we get morphisms

$$
Z_{i}: p_{i}^{*} \mathcal{V} \rightarrow V^{\gamma_{i}} \otimes \mathcal{O}_{\mathcal{S}}
$$

Hence, by adjunction we can extend $\mathcal{V}$ to an object

$$
[\mathcal{V}, \Sigma]:=\left(\mathcal{V},\left(V^{\gamma_{i}} \otimes \mathcal{O}_{\mathcal{S}}\right),\left(Z_{i}\right)\right)
$$

of $\operatorname{Coh}(\mathcal{C}, \Sigma)$.

On the other hand, we can combine $\chi_{P}$ with $W$ into a polynomial morphism

$$
W_{\mathcal{V}}: \mathcal{V}=\mathcal{P} \times_{\Gamma} V \rightarrow \omega_{\mathcal{C} / \mathcal{S}}^{\log }:(x, v) \mapsto W(v) \cdot \chi_{P}(x)
$$

We can view it as a linear morphism of vector bundles on $\mathcal{C}$,

$$
W_{\mathcal{V}}: S^{\bullet}(\mathcal{V})_{d} \rightarrow \omega_{\mathcal{C} / \mathcal{S}}^{\log }
$$

where we grade the symmetric algebra of $\mathcal{V}$ using the $\mathbb{G}_{m, R}$-action on $\mathcal{V}$. Furthermore, this morphism is compatible with the morphisms (3.2.1), so that the following diagram is commutative

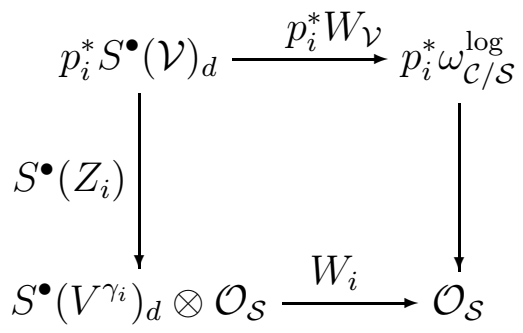

where $W_{i}=\left.W\right|_{V^{\gamma_{i}}}$. This means that we have a morphism

$$
\left(W_{\mathcal{V}},\left(W_{i}\right)\right): S^{\bullet}[\mathcal{V}, \Sigma]_{d} \rightarrow\left[\omega_{\mathcal{C} / \mathcal{S}}^{\log }, \Sigma\right]
$$

in the category $\mathrm{Q} \operatorname{coh}(\mathcal{C}, \Sigma)$ (where again we take the part of weight $d$ with respect to $\left.\mathbb{G}_{m, R}\right)$. Next, we can take the derived push-forward with respect to the morphism of pairs (2.2.1). Together with (2.1.1) this gives us a morphism

$$
S^{\bullet}\left(R \pi_{*}[\mathcal{V}, \Sigma]\right)_{d} \rightarrow R \pi_{*} S^{\bullet}[\mathcal{V}, \Sigma]_{d} \rightarrow R \pi_{*}\left[\omega_{\mathcal{C} / \mathcal{S}}^{\log }, \Sigma\right]
$$


in $D \operatorname{Qcoh}(\mathcal{S}, \mathcal{S})$.

Now let us set

$$
E:=R j ! S^{\bullet}\left(R \pi_{*}[\mathcal{V}, \Sigma]\right)_{d} .
$$

Applying $R j^{\text {! }}$ to morphism (3.2.3), we obtain a morphism

$$
E=R j^{!} S^{\bullet}\left(R \pi_{*}[\mathcal{V}, \Sigma]\right)_{d} \rightarrow R j^{!} R \pi_{*}\left[\omega_{\mathcal{C} / \mathcal{S}}^{\log }, \Sigma\right] \simeq R \pi_{*} \omega_{\mathcal{C} / \mathcal{S}},
$$

where the last isomorphism is (2.2.2). It is easy to see that it fits into a morphism of exact triangles

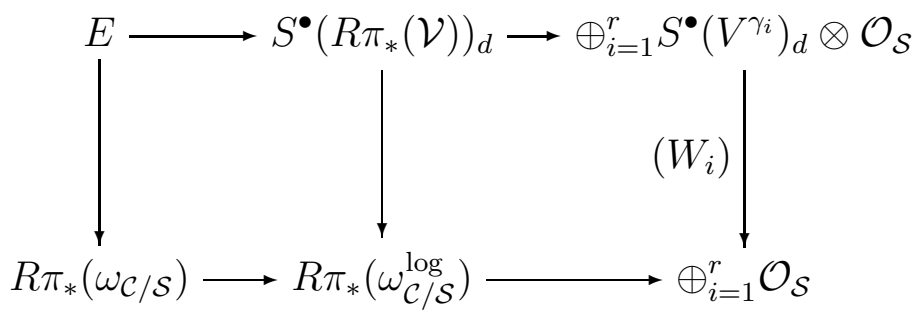

Combining it with the morphism of triangles (2.2.3), we get a commutative diagram with the exact triangle in the first row

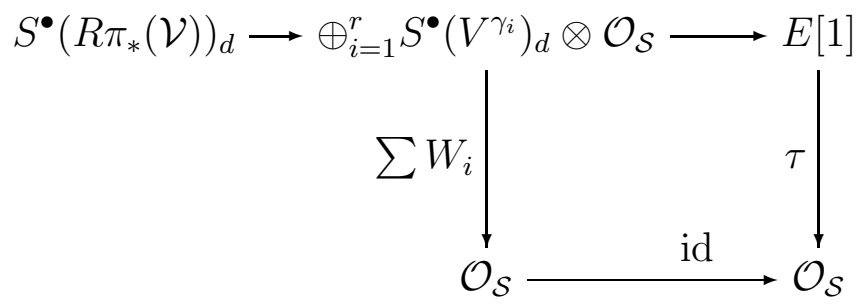

Dualizing we get a commutative diagram

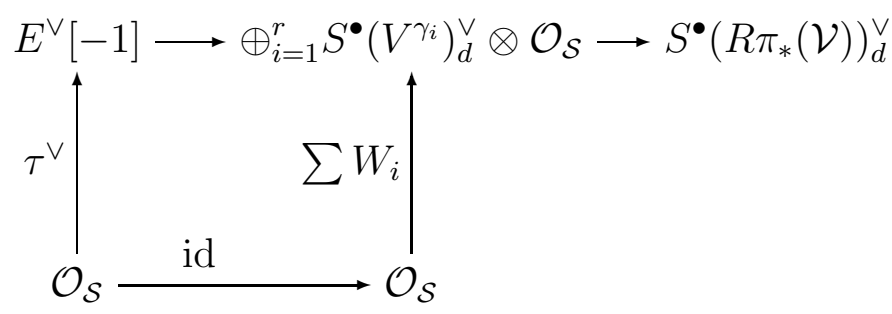

This implies that the pull-back $Z^{*}\left(\bigoplus_{i} W_{i}\right)$ with respect to the morphism

$$
Z:\left[R \pi_{*}(\mathcal{V})\right] \rightarrow \prod_{i} V^{\gamma_{i}}
$$

induced by (3.2.1), becomes zero in cohomology of the structure sheaf on $\left[R \pi_{*}(\mathcal{V})\right]$.

In fact, we can realize this functon by an explicit coboundary. For this we need a realization of the above diagram in the homotopy category of complexes. As in [6, Sec. $4.2]$, the starting point is that $R \pi_{*}(\mathcal{V})$ can realized $\left(\mathbb{G}_{m, R^{-}}\right.$equivariantly) by a complex of the form $[A \rightarrow B]$ in such a way that the morphism (3.2.5) is realized by a surjective 
morphism $A \rightarrow \bigoplus_{i=1}^{r} V^{\gamma_{i}} \otimes \mathcal{O}_{S}$. Then the first line of the diagram (3.2.4) can be realized by a short exact sequence of complexes

$$
0 \rightarrow \operatorname{ker}\left(S^{\bullet}(Z)_{d}\right) \rightarrow S^{\bullet}(A \rightarrow B)_{d} \stackrel{S^{\bullet}(Z)_{d}}{\longrightarrow} \bigoplus_{i=1}^{r} S^{\bullet}\left(V^{\gamma_{i}}\right)_{d} \otimes \mathcal{O}_{S} \rightarrow 0
$$

where the complex $S^{\bullet}(A \rightarrow B)_{d}$, concentrated in degrees $[0, \operatorname{rk}(B)]$, has form

$$
S^{\bullet}(A)_{d} \rightarrow\left(S^{\bullet}(A) \otimes B\right)_{d} \rightarrow\left(S^{\bullet}(A) \otimes \wedge^{2} B\right)_{d} \rightarrow \ldots
$$

Using this we get a canonical quasi-isomorphism of $E$ with the bounded complex of vector bundles

$$
K^{\bullet}:=\operatorname{Cone}\left(S^{\bullet}\left(R \pi_{*}(\mathcal{V})\right)_{d} \rightarrow \oplus_{i=1}^{r} S^{\bullet}\left(V^{\gamma_{i}}\right)_{d} \otimes \mathcal{O}_{\mathcal{S}}\right)[-1] .
$$

Now we want to realize the morphism $\tau: E \rightarrow \mathcal{O}_{S}[-1]$ in the derived category by a morphism $K^{\bullet} \rightarrow \mathcal{O}_{S}[-1]$ in the homotopy category of complexes.

By changing $[A \rightarrow B]$ to a quasi-isomorphic complex $[\bar{A} \rightarrow \bar{B}]$ one can achieve that for $i \geq 1$ the terms $K^{i}$ satisfy $\operatorname{Ext}^{>0}\left(K^{i}, \mathcal{O}_{S}\right)=0$ (see [6, Lem. 4.2.5]). This implies that morphisms $K \rightarrow \mathcal{O}_{S}[-1]$ in the homotopy category of complexes and in the derived category are the same.

The dual of this morphism can be interpreted as a canonical homotopy (up to a homotopy between homotopies) $f_{-1}$ between the function $Z^{*}\left(\bigoplus_{i} W_{i}\right)$ on $\left[R \pi_{*} \mathcal{V}\right]$ and 0 . As we have seen in Example 1.1.1, this corresponds to a structure $\delta=d-f_{-1} \cdot$ id of a dg-matrix factorization of $-Z^{*}\left(\bigoplus_{i} W_{i}\right)$ on the structure sheaf of $\left[R \pi_{*} \mathcal{V}\right]$.

Furthermore, it carries an equivariant structure with respect to the action of the center $Z(\Gamma)$ of $\Gamma$ (acting trivially on the base) and with respect to $\prod_{i} C_{G}\left(\gamma_{i}\right)$ (changing the rigidifications).

3.3. Properties. The first important property is that our dg-matrix factorization over $\left[R \pi_{*} \mathcal{V}\right]$ is supported on the zero section in $\left[R \pi_{*} \mathcal{V}\right]$. Since each $W_{i}$ is non-degenerate, we know that the support belongs to the zero locus of $Z^{*}\left(\bigoplus_{i} W_{i}\right)$. Thus, we reduce to considering the following situation. Let $C$ be a curve, $\mathcal{V}$ be a vector bundle over $C$, equipped with a $\mathbb{G}_{m}$-equivariant structure (where $\mathbb{G}_{m}$ acts trivially on $C$ ). Assume also we have a polynomial morphism $W_{\mathcal{V}}: \mathcal{V} \rightarrow \omega_{C}$, homogeneous of degree $d$, such that over an open dense subset of $C$ there exists a trivialization $\mathcal{V} \simeq V \otimes \mathcal{O}_{C}$ (compatible with the $\mathbb{G}_{m}$-action) such that $W_{\mathcal{V}}$ is induced by our polynomial $W$ on $V$. Then we have the induced polynomial function of degree -1 on the dg-affine space $\left[H^{0}(C, \mathcal{V}) \oplus\right.$ $\left.H^{1}(C, \mathcal{V})[-1]\right]$, induced by $W_{\mathcal{V}}$ and by the identification $H^{1}\left(C, \omega_{C}\right) \simeq \mathbb{C}$. We claim that it is supported at the origin. Indeed, we start by observing that the preimage of the origin under the gradient morphism $\Delta W: V \rightarrow V^{\vee}$ is still the origin (since $W$ is nondegenerate). From this we get the similar assertion about the preimage of the zero section under the relative gradient morphism $\Delta W_{\mathcal{V}}: \mathcal{V} \rightarrow \mathcal{V}^{\vee} \otimes \omega_{C}$. Finally, we note that the support of our function on $\left[H^{0}(C, \mathcal{V}) \oplus H^{1}(C, \mathcal{V})[-1]\right]$ coincides with the vanishing locus of the polynomial morphism

$$
H^{0}(C, \mathcal{V}) \rightarrow H^{0}\left(\mathcal{V}^{\vee} \otimes \omega_{C}\right) \simeq H^{1}(C, \mathcal{V})^{\vee}
$$

induced by the relative gradient map. This implies our claim. 
Next, the key gluing property satisfied by the fundamental matrix factorizations (cf. [6, Sec. 5.2, 5.3]) holds in the situation when we consider two natural families of orbicurves $\widetilde{C} \stackrel{\widetilde{\pi}}{\longrightarrow} S, C \stackrel{\pi}{\longrightarrow} S$, over

$$
S:=S_{g_{1}}^{\mathrm{rig}}\left(\gamma_{1}, \ldots, \gamma_{n_{1}}, \gamma\right) \times S_{g_{2}}^{\mathrm{rig}}\left(\gamma_{1}^{\prime}, \ldots, \gamma_{n_{2}}^{\prime}, \gamma^{-1}\right),
$$

where $\widetilde{C}$ is the disconnected curve and $C$ is obtained by gluing two points into a node (there is also a similar picture corresponding to a non-disconnecting node). We denote by $f: \widetilde{C} \rightarrow C$ the gluing morphism.

In this setting there are natural $\Gamma$-spin structures $\widetilde{P}$ (resp., $P$ ) over $\widetilde{C}$ (resp., $C$ ), where $P$ is obtained by gluing fibers of $\widetilde{P}$ over the two points that are glued into a node, using the rigidifications and the square root of $J, J^{1 / 2} \in \mathbb{G}_{m, R}$ such that $\chi\left(J^{1 / 2}\right)=-1$ (see $[6$, Sec. 5.2]). The main compatibility between the push-forwards of the corresponding vector bundles $\widetilde{\mathcal{V}}$ and $\mathcal{V}$ is given by the cartesian diagram

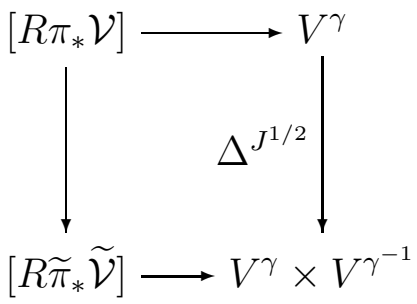

where $\Delta^{J^{1 / 2}}: V^{\gamma} \rightarrow V^{\gamma} \times V^{\gamma^{-1}}$ is the twisted diagonal map: $x \mapsto\left(x, J^{1 / 2} x\right)$. Furthermore, the natural dg-matrix factorization on $\left[R \pi_{*} \mathcal{V}\right]$ is identified with the pull-back of the one on $\left[R \widetilde{\pi}_{*} \widetilde{\mathcal{V}}\right]$.

Recall that in [6], in the case when $G$ is contained in an algebraic torus acting on $V$, we used the fundamental matrix factorizations to construct cohomological field theories associated with $(W, G)$ by viewing them as kernels for Fourier-Mukai functors and passing to Hochschild homology. Similarly, in the case of nonabelian $G$ one can use the above construction to get a cohomological field theory (with coefficients in $\mathbb{C}$ ) and show some of the properties predicted in [4]. We will study the resulting cohomological field theories elsewhere.

\section{REFERENCES}

[1] I. Ciocan-Fontanine, M. Kapranov, Virtual fundamental classes via dg-manifolds, Geom. Topol. 13 (2009), 1779-1804.

[2] A. I. Efimov, L. Positselski, Coherent analogues of matrix factorizations and relative singularity categories, Algebra Number Theory 9 (2015), 1159-1292.

[3] H. Fan, T. Jarvis, Y. Ruan, The Witten equation, mirror symmetry, and quantum singularity theory, Ann. of Math. (2) 178 (2013), 1-106.

[4] H. Fan, T. Jarvis, Y. Ruan, A mathematical theory of the gauged linear sigma model, Geom. Topol. 22 (2018), 235-303.

[5] V. Lunts, Categorical resolutions, poset schemes, and Du Bois singularities, IMRN 19 (2012), 43724420.

[6] A. Polishchuk, A. Vaintrob, Matrix factorizations and cohomological field theories, J. Reine Angew. Math. 714 (2016), 1-122. 